\title{
Building up the tension between the epithelial and stromal compartment in pancreatic ductal adenocarcinoma
}

\author{
G Biffi', D Öhlund ${ }^{1}$ and D Tuveson ${ }^{*, 1}$
}

Cell Death and Differentiation (2016) 23, 1265-1266; doi:10.1038/cdd.2016.50; published online 10 June 2016

In recent years, pancreatic cancer-associated stroma has been the topic of a number of studies that have proposed stromal components as promising, but still controversial, targets for novel therapeutic strategies. A new study describes how epithelial TGF- $\beta$ signalling deficiency and consequential STAT3 activation act as key players in modulating the desmoplastic reaction, with implications for both clinical outcome and future drug development. ${ }^{1}$

Pancreatic ductal adenocarcinoma (PDAC) is characterised by a preponderant stromal compartment that largely exceeds the epithelial component. The effects of PDAC stroma on the pathophysiology of the disease go beyond the stroma acting as a barrier to drug delivery. ${ }^{2-4}$ Indeed, PDAC stroma has largely been described as a supporting niche that favours tumour progression ${ }^{5,6}$ and immune cell exclusion, ${ }^{7}$ and causes resistance to common chemotherapeutic agents, ${ }^{8}$ thus representing a potential target for therapeutic intervention. Nonetheless, the concept of tumour stroma as a tumour-promoting entity has been challenged by recent studies that aimed to selectively target stromal components and showed that depletion of specific stromal cell populations or blocking of stromal signalling pathways could result in worse outcomes. 9,10 Importantly, these studies underline a high degree of complexity within the tumour stroma, and highlight the fact that targeting of certain stromal components can indeed be more deleterious than helpful. These results call for a deeper understanding of the composition of this multifaceted stromal landscape, of the underlying mechanisms that drive the desmoplastic reaction, and of its pathophysiological consequences. Such analysis will clarify which components within the stroma are suitable therapeutic targets and which are not.

The study from Laklai et al. ${ }^{1}$ adds to this discussion by providing evidence that specific features of both the epithelial compartment, such as actomyosin tension, and of the adjacent stroma, such as presence of certain fibrillar collagens, abundance of alpha-smooth muscle actin ( $\alpha$ SMA)-positive stromal cells, and thickness of collagen fibres are associated with various degrees of human PDAC differentiation status and patient prognosis. The authors further show that mutations in a specific pathway in the epithelial compartment in both human PDAC and mouse models are responsible for inducing changes in the tumour microenvironment. Indeed, loss-of-function mutations in the epithelial transforming growth factor $\beta$ (TGF- $\beta$ ) signalling pathway enhance the activity of signal transducer and activator of transcription 3 (STAT3). As a consequence, STAT3 activity leads to increased epithelial tension and contractility, and alters the adjacent stroma by heightening tissue inflammation and fibrosis with formation of thicker collagen fibres and a stiffer extracellular matrix. The authors further show that this altered stromal architecture in turn can induce changes in the adjacent epithelium, as shown by accelerated PDAC progression and consequent decreased survival (Figure 1). STAT3 depletion or inhibition blocks these effects, confirming that increased epithelial tension and reprogramming of the adjacent stroma promote tumour progression. Thus, these new findings unveil a novel epithelial - stromal crosstalk with profound implications for tumour composition and progression. In light of this study and previous results that highlighted the role of STAT3 in PDAC development, ${ }^{11}$ the identification of any pathways that stimulate STAT3 signalling in PDAC may reveal additional therapeutic opportunities.

It is now evident that the tumour stroma conceals elements that are either tumour-promoting or tumour-inhibiting. ${ }^{12}$ Laklai et al. ${ }^{1}$ show that, regardless of the composition of the bulk stroma, changes in one signalling pathway within the PDAC epithelium are enough to increase tumour cell tension and induce a stiffer, matricellular-enriched fibrosis, thereby reprogramming the adjacent stroma with a more tumour-promoting phenotype. The new findings not only stress the fundamental role of tumour - stroma crosstalk in PDAC progression, but also give ideas for novel stroma-targeting therapeutic strategies.

\section{Conflict of Interest}

The authors declare no conflict of interest.

Acknowledgements. GB is supported by the Human Frontiers Science Program (LT000195/2015- L) and the European Molecular Biology Organization (EMBO, ALTF 1203-2014). DÖ is funded by The Swedish Research Council (5372013-7277), the Västerbotten County Council, The Kempe Foundations (JCK-1301), and the Swedish Society of Medicine (SLS-326921, SLS-250831). DT is a distinguished scholar of the Lustgarten Foundation and Director of the Lustgarten Foundation-designated Laboratory of Pancreatic Cancer Research. DT is also

\footnotetext{
${ }^{1}$ The Cancer Centre at Cold Spring Harbor Laboratory (CSHL), New York, NY, USA

*Corresponding author: D Tuveson, The Cancer Centre at Cold Spring Harbor Laboratory (CSHL), New York, NY, USA. Tel: +1 5163675246 ; Fax: +1 5163678353 ; E-mail: dtuveson@cshl.edu
} 


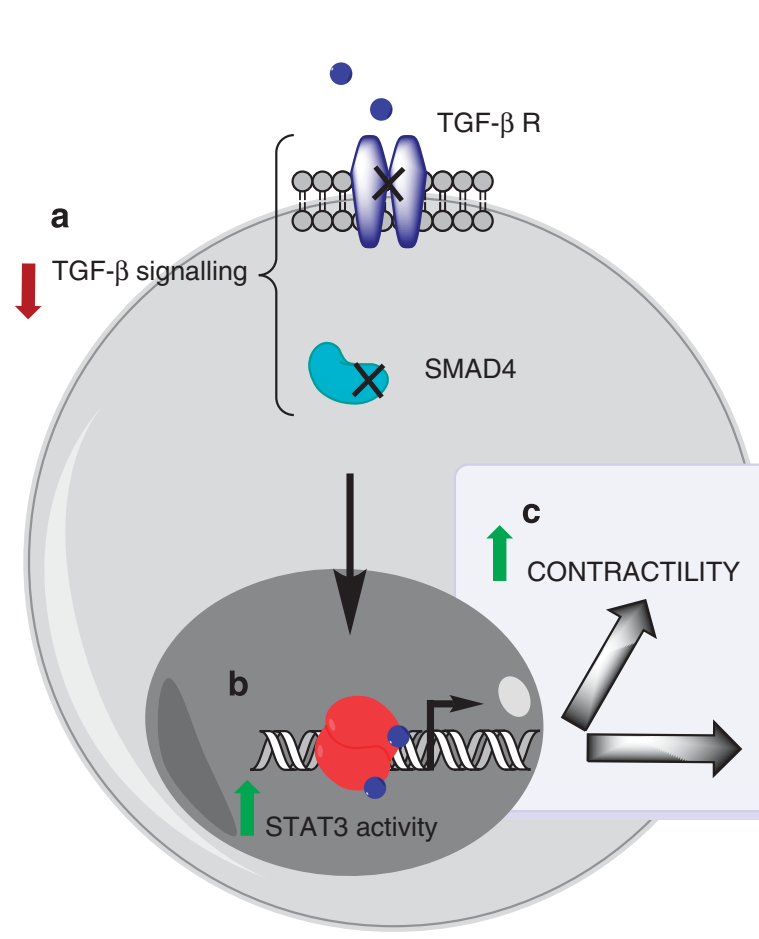

X Loss-of-function mutation

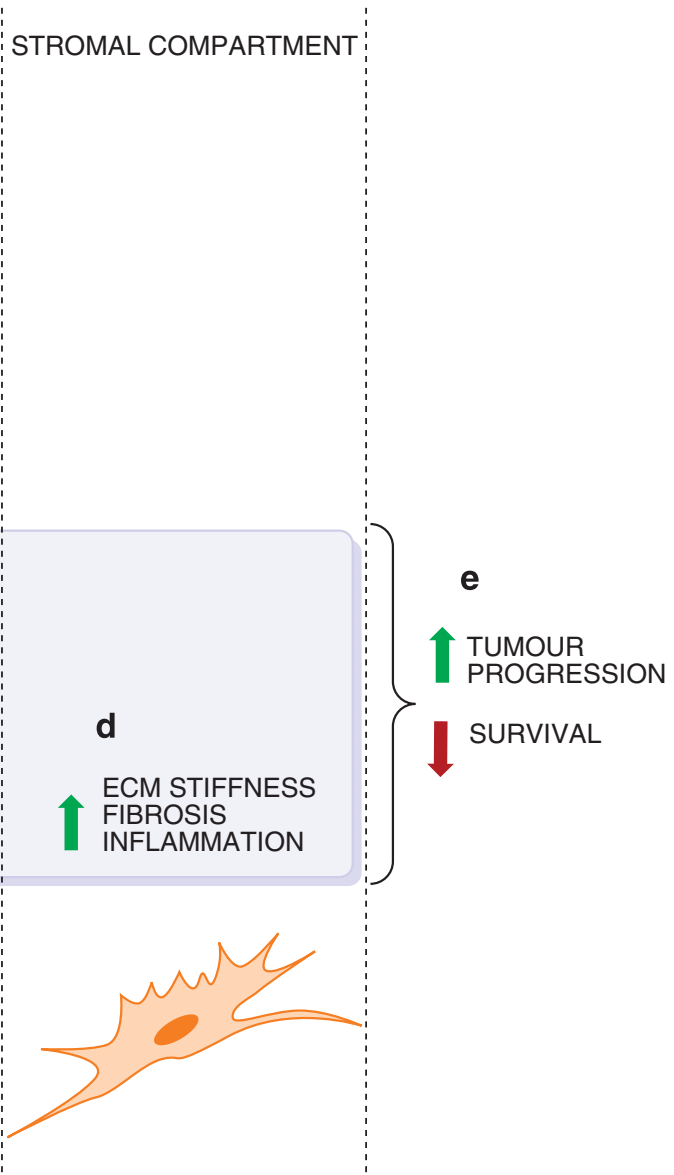

Figure 1 Role of epithelial STAT3 in driving stromal remodelling and tumour tension. Loss-of-function mutations in the TGF- $\beta$ signalling pathway, either at the receptor level or further downstream (SMAD4), lead to impaired TGF- $\beta$ signalling (a). This specific genetic signature results in increased STAT3 activity (b), leading to both cancer cell autonomous and nonautonomous effects. Within the epithelial compartment, phosphorylation of actomyosins causes increased contractility and tumour tension (c), whereas epithelial STAT3 activation models the surrounding stroma by increasing fibrosis and ECM stiffness, and modulating immune cell infiltration (d). Altogether, these epithelial and stromal changes drive tumour progression and lead to shorter survival (e)

supported by the Cancer Center Support Grant 5P30CA045508, the Cold Spring Harbor Laboratory Association, the NIH (5P30CA45508-26, 5P50CA101955-07, 1U10CA180944-01, 5U01CA168409-3, and 1R01CA190092-01), the Carcinoid Foundation, the David Rubinstein Center for Pancreatic Cancer Research at MSKCC, Stand Up to Cancer/KWF (DT), the STARR foundation (17-A718), and DOD (W81XWH-13-PRCRP-IA).

1. Laklai $\mathrm{H}$ et al. Nat Med 2016 (this issue).

2. Olive KP et al. Science 2009; 324: 1457-1461.
3. Jacobetz MA et al. Gut 2012 (this issue).

4. Provenzano PP et al. Cancer Cell 2012; 21: 418-429.

5. Neesse $A$ et al. Gut 2011; 60: 861-868.

6. Hwang RF et al. Cancer Res 2008; 68: 918-926.

7. Feig $C$ et al. Proc Natl Acad Sci USA 2013; 110: 20212-20217.

8. Miyamoto $\mathrm{H}$ et al. Pancreas 2004; 28: 38-44.

9. Ozdemir BC et al. Cancer Cell 2014; 25: 719-734.

10. Rhim AD et al. Cancer Cell 2014; 25: 735-747.

11. Corcoran RB et al. Cancer Res 2011; 71: 5020-5029.

12. Ohlund D et al. J Exp Med 2014; 211: 1503-1523. 